\title{
Search for Critical Slip Surface in Slope Stability Analysis by Spline-Based GA Method
}

\author{
Jianping Sun'; Jiachun $\mathrm{Li}^{2}$; and Qingquan $\mathrm{Liu}^{3}$
}

\begin{abstract}
The stability of a soil slope is usually analyzed by limit equilibrium methods, in which the identification of the critical slip surface is of principal importance. In this study the spline curve in conjunction with a genetic algorithm is used to search the critical slip surface, and Spencer's method is employed to calculate the factor of safety. Three examples are presented to illustrate the reliability and efficiency of the method. Slip surfaces defined by a series of straight lines are compared with those defined by spline curves, and the results indicate that use of spline curves renders better results for a given number of slip surface nodal points comparing with the approximation using straight line segments.
\end{abstract}

DOI: 10.1061/(ASCE)1090-0241(2008)134:2(252)

CE Database subject headings: Slope stability; Safety; Algorithms; Limit equilibrium.

\section{Introduction}

Determination of the critical slip surface is a central issue to slope stability analysis. Previous research has been devoted to locating the critical slip surface. Earlier works employed the variational calculus (Baker and Gaber 1978) and the dynamic programming (Baker 1980). Celestino and Duncan (1981) and Li and White (1987) utilized alternating variable methods to search for the critical noncircular slip surface. Greco (1996) and Malkawi et al. (2001) used the Monte Carlo technique to determine the critical slip surface. In recent years, Goh (1999), McCombic and Wilkinson (2002), Das (2005), and Zolfaghari et al. (2005) successfully introduced the genetic algorithm (GA) into slope stability analysis for critical surface identification.

In general, a potential slip surface is divided into segments by a number of nodal points, and each pair of contiguous nodal points is connected by a straight line (Baker 1980; Greco 1996; Malkawi et al. 2001) or a smooth curve. The smooth curve may be generated by splines (Donald and Chen 1997). In this study a combination of spline curves and GA is used to search for the critical slip surface, and Spencer's method is employed to calculate the factor of safety.

${ }^{1}$ Doctoral Student, Institute of Mechanics, Chinese Academy of Sciences, Beijing 100080, China. E-mail: sunjianp@mails.gucas.ac.cn

${ }^{2}$ Professor, Institute of Mechanics, Chinese Academy of Sciences, Beijing 100080, China. E-mail: jcli05@imech.ac.cn

${ }^{3}$ Professor, Institute of Mechanics, Chinese Academy of Sciences, Beijing 100080, China. E-mail: qqliu@imech.ac.cn

Note. Discussion open until July 1, 2008. Separate discussions must be submitted for individual papers. To extend the closing date by one month, a written request must be filed with the ASCE Managing Editor. The manuscript for this technical note was submitted for review and possible publication on May 26, 2006; approved on June 21, 2007. This technical note is part of the Journal of Geotechnical and Geoenvironmental Engineering, Vol. 134, No. 2, February 1, 2008. @ASCE, ISSN $1090-0241 / 2008 / 2-252-256 / \$ 25.00$.

\section{Determination of Slip Surface}

A slip surface is represented by $n$ nodal points with coordinates $\left(x_{1}, y_{1}\right),\left(x_{2}, y_{2}\right), \ldots,\left(x_{n}, y_{n}\right)$, respectively in the $x-y$ plane [Fig. 1(a)]. In order to minimize the number of variables, any two contiguous nodal points maintain the same horizontal distance, such that

$$
x_{i}=x_{i-1}+\left(x_{n}-x_{1}\right) /(n-1) \quad \text { for } i=2, n-1
$$

The abscissas of all nodal points should be enclosed within $x_{\min }$ and $x_{\max }$, and accordingly

$$
x_{\min } \leqslant x_{i} \leqslant x_{\max } \text { for } i=1, n
$$

Also, $y_{1}$ and $y_{n}$ are related to the topographic profile $s(x)$, and

$$
y_{i}=s\left(x_{i}\right) \quad \text { for } i=1 \quad \text { and } \quad i=n
$$

As a result, a specific slip surface can be expressed mathematically by an $n$-element array

$$
S=\left[x_{1}, y_{2}, y_{3}, \ldots, y_{n-1}, x_{n}\right]^{T}
$$

where $T=$ transposed symbol.

The objective function locating the critical slip surface, which is defined as a surface with the minimum factor of safety among all the available ones, can be stated

$$
\min F(S)
$$

To satisfy the requirements of kinematic admissibility, these segments, defined by any two contiguous nodal points, are further assumed to be concave upward which means that

$$
\alpha_{1} \leqslant \alpha_{2} \leqslant \cdots \leqslant \alpha_{i} \leqslant \cdots \leqslant \alpha_{n-1}
$$

where $\alpha_{i}$, the inclination of segments, must range between -45 and $60^{\circ}$ avoid computational divergence encountered in calculating the safety factor (Malkawi et al. 2001). 

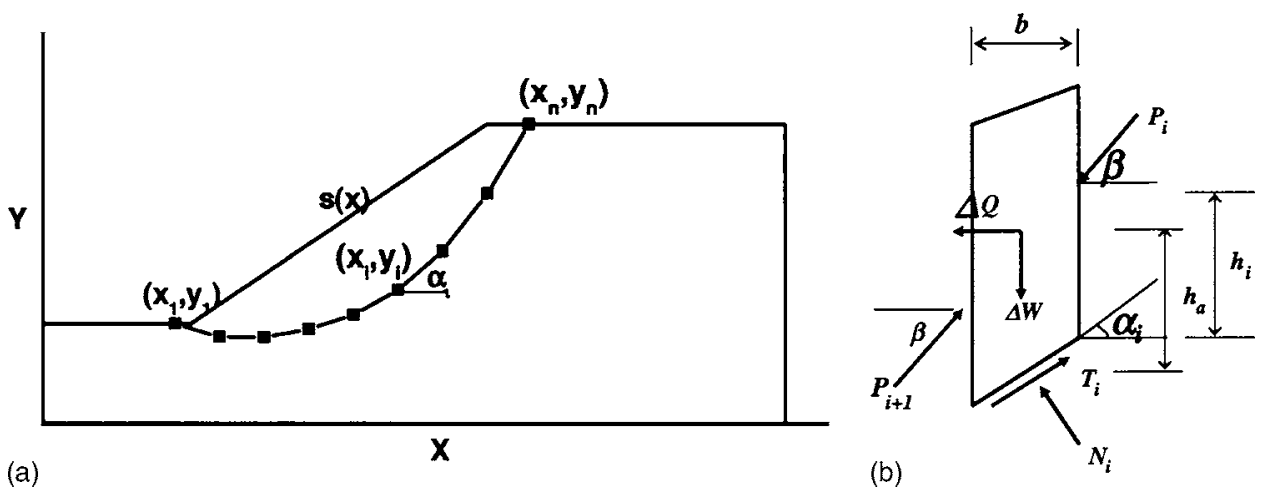

Fig. 1. General cross section of (a) slope; (b) forces acting on a typical slice

Subsequently, the nodal points are connected by cubic-spline interpolation. The cubic-spline interpolation is to construct a piecewise cubic polynomial function of the dependent variable between two nodal points such that the interpolation function must pass through each nodal point and be continuous in its first and second derivatives at any interior nodal point. Then, the soil mass above the created slip surface is divided into a number of vertical slices. If there is a weak layer in the slope, intuitively the critical slip surface should extend along the weak layer for a substantial length. If the abscissa of any slice base is lower than the weak layer, this slice base is adjusted to the weak layer.

\section{Calculation of Safety Factor}

Spencer's method (1967) is used to calculate the safety factor. Interslice forces are included by assuming the interslice force inclination angles of all slices to be equal. Both force and moment equilibrium are satisfied explicitly. Spencer's method is applicable to slip surfaces of any shape, and is considered to be one of the most accurate methods for slope stability analysis (Duncan and Wright 1980). Fig. 1(b) shows the details of interslice forces for a typical slice.

The equations of force and momentum equilibrium can be respectively written as

$$
P_{i}=P_{i+1}+\frac{F \Delta W \sin \alpha_{i}-c^{\prime} b \sec \alpha_{i}-\Delta W \cos \alpha_{i} \tan \varphi^{\prime}+U_{i} b \sec \alpha_{i} \tan \varphi^{\prime}+\Delta Q\left(F-\tan \varphi^{\prime} \tan \alpha_{i}\right) \cos \alpha_{i}}{\sin \left(\beta-\alpha_{i}\right) \tan \varphi^{\prime}-F \cos \left(\beta-\alpha_{i}\right)}
$$

$$
\begin{aligned}
h_{i}= & \frac{P_{i+1}}{P_{i}} h_{i+1}-\frac{P_{i+1}}{P_{i}} \frac{b}{2} \tan \alpha_{i}+\frac{b}{2} \tan \beta+\frac{P_{i+1}}{P_{i}} \frac{b}{2} \tan \beta \\
& -\frac{\Delta Q h_{a}}{P_{i} \cos \beta}-\frac{b}{2} \tan \alpha_{i}
\end{aligned}
$$

where $F$ =factor of safety and $P_{i}$ and $P_{i+1}=$ right and the left interslice force, respectively. The total normal force and the pore water pressure on the slice base are $N_{i}$ and $U_{i}$, respectively. The weight of slice is $\Delta W$ and $\Delta Q=$ horizontal force of slice. The angle between the slice base and the horizontal line is $\alpha_{i}$, and $\beta=$ inclination angle of interslice force. The height of force $P_{i}$ and the center of the slice are $h_{i}$ and $h_{a}$, respectively, and $b=$ width of the slice.

\section{Genetic Algorithm}

Basically, GA (Goh 1999) is based on Darwin's theory of survival of the fittest, which is based on the principle that solutions to a problem can be obtained through evolution. The algorithm starts with a set of possible solutions. The set of possible solutions is called the "population." Each possible solution within the population is called a "chromosome." Associated with each chromosome is a fitness value, which is found by evaluating the chromosome with respect to the objective function. Solutions from one population are taken and used to construct a new population so that the new population (offspring) will be fitter than the old one. This process is repeated until the best chromosome representing the optimum solution is produced or some termination criterion, such as a set number of generations is reached. The fundamentals of GA are described in the following sections.

\section{Fitness Function}

As GA only allows for optimization to find a maximum value, the fitness function is defined as $f_{\max }=1 / F_{\min }$ in slope stability analysis, where $F_{\min }=$ minimum factor of safety. 


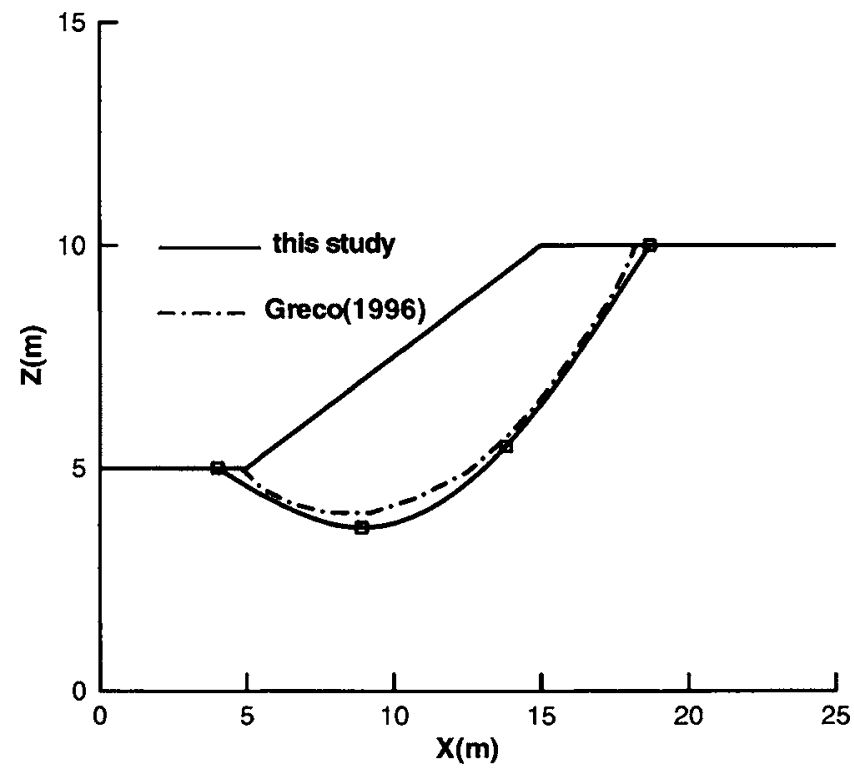

Fig. 2. Cross section of slope of Example 1. The solid line represents the critical slip surface of this study, and the broken line is the solution given by Greco (1996, ASCE).

\section{Initialization}

The genetic algorithm starts with a population of $N$ possible solutions. Each solution is created by $n$ binary encoding, $\beta$, which is a number drawn from the period of $[0,1]$. The $\beta_{i}$ generation mainly depends on a function that generates sets of random numbers. These sets of random numbers depend on the "iseed" number that is used in the function, $\beta_{i}$ along with all the random numbers corresponding to the iseed number changes. The generation procedure with different iseed numbers is repeated to insure that the critical slip surface is reached. The relation between $\beta_{i}(i=1, \ldots, n)$ and $\left[x_{1}, y_{2}, \ldots, y_{n-1}, x_{n}\right]^{T}$ can be described as follows:

$$
\begin{gathered}
x_{1}=x_{\min }+\beta_{1}\left(x_{\max }-x_{\min }\right) \\
x_{n}=x_{1}+\beta_{n}\left(x_{\max }-x_{1}\right) \\
y_{i}=y_{\text {ilow }}+\beta_{i}\left(y_{\text {iupp }}-y_{\text {ilow }}\right)
\end{gathered}
$$

where $y_{\text {iupp }}$ and $y_{\text {ilow }}$ are defined by considering the slip surface to be kinematically admissible.

Table 1. Minimum Safety Factor Given by Minimization Procedures for Example 1

\begin{tabular}{lcc}
\hline Source & Method & $\begin{array}{c}\text { Range of } \\
\text { safety factor }\end{array}$ \\
\hline Yamagami and Ueta (1988) & BFGS & 1.338 \\
& DFP & 1.338 \\
& Powell & 1.338 \\
Greco (1996) & Simplex & $1.339-1.348$ \\
This study & Pattern search & $1.326-1.330$ \\
& Monte Carlo & $1.327-1.333$ \\
& Genetic algorithm & 1.324 (line) \\
& & 1.321 (spline) \\
\hline
\end{tabular}

Table 2. Effect on the Safety Factor from Varying the Number of Slices for Example 1

\begin{tabular}{lllccc}
\hline Number of slices & 8 & 16 & 32 & 64 & 128 \\
\hline Factor of safety & 1.225 & 1.294 & 1.318 & 1.321 & 1.321 \\
Percentage error & 0.07 & 0.02 & 0.002 & $<0.0001$ & $<0.0001$ \\
\hline
\end{tabular}

\section{Selection}

The selection operator is used to determine which chromosomes are chosen as parents that will create offspring for the next generation. In this study, the roulette wheel selection is used in conjunction with elitism. This usually involves retaining the best chromosome at each generation to ensure that the best chromosome is not lost if it is not selected in reproduction or if it is destroyed by crossover or mutation.

\section{Crossover and Mutation}

The single-point crossover operator is employed in this study and the randomly flipping mutation procedure is used.

\section{Numerical Applications}

In order to assess the performance of the spline curve in finding the critical slip surface, three examples were selected from the literature. The number of slices used is 50 for all examples presented in this study, and there is no relationship between the number of nodal points and the number of slices in the computations. Factors of safety from analyses are given to the three decimal places for the purpose of comparison, but such high resolution is never required in practice. Failure surfaces defined by straight lines are compared with those defined by spline curves. The number of nodal points used range from 4 to 8 . Parameters for GA are chosen in these examples as follows: The number of generations is 300 , the population size is 20 , the cross-over rate is 0.85 , and the mutation rate is 0.02 .

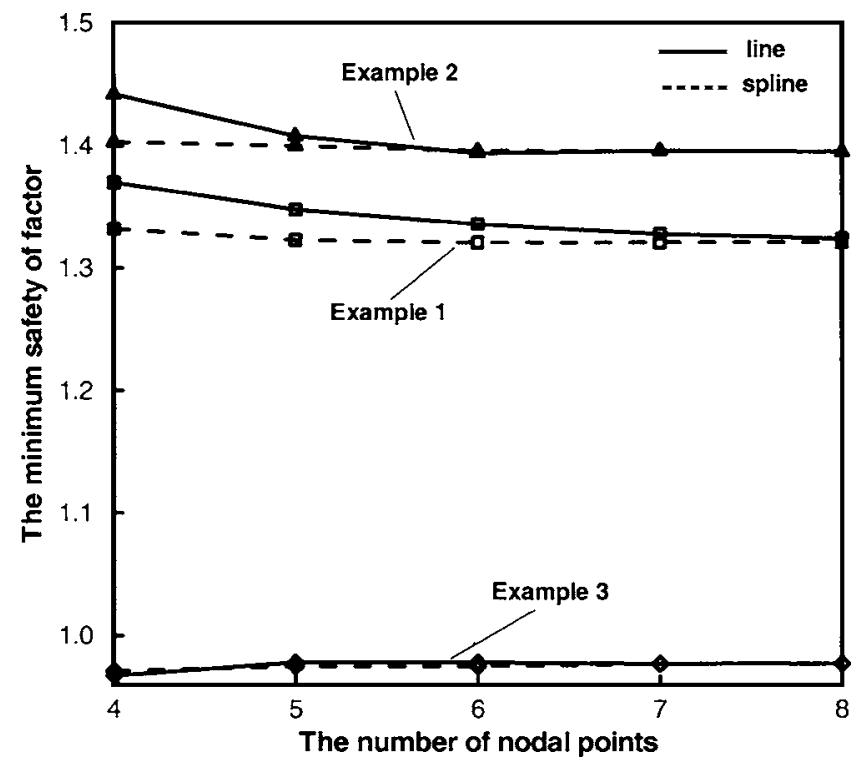

Fig. 3. Factor of safety versus the number of nodal points 


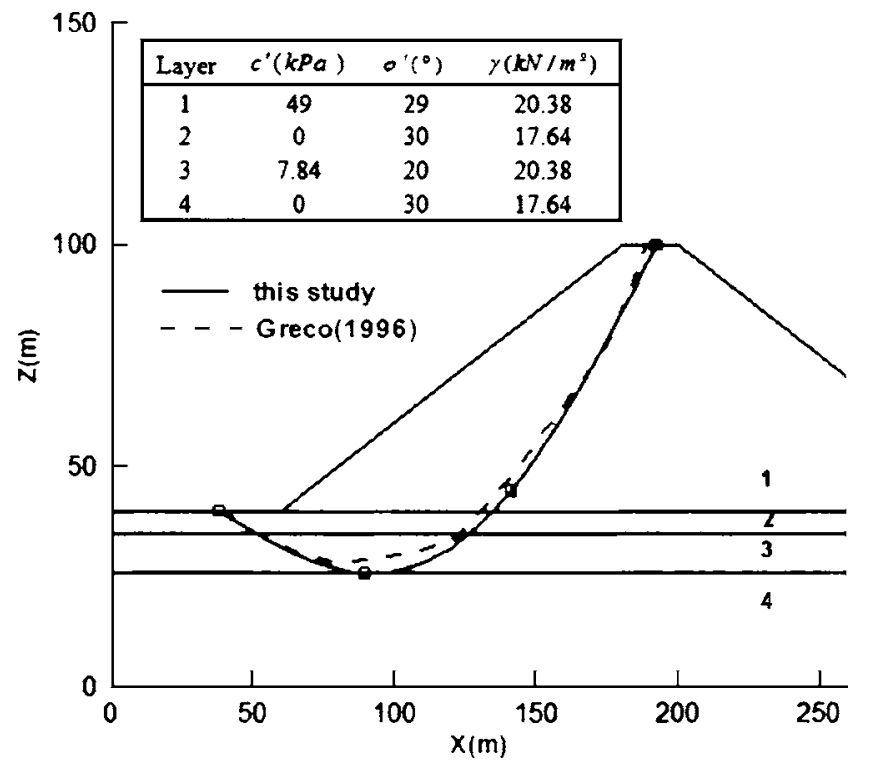

Fig. 4. Cross section of slope of Example 2. The solid line represents the critical slip surface of this study, the broken line is the solution given by Greco (1996, ASCE).

\section{Example 1, Homogeneous Slope}

This example is of a homogeneous slope (Fig. 2) with $\gamma=17.64 \mathrm{kN} / \mathrm{m}^{3}, \phi^{\prime}=10^{\circ}$ and $c^{\prime}=9.8 \mathrm{kPa}$. Yamagami and Ueta (1988) utilized nonlinear programming methods, which include the DFP method as suggested by Davidon and modified by Fletcher and Powell (Malkawi et al. 2001), the BFGS method proposed by Broyden, Fletcher, Goldfarb, and Shanno (Malkawai et al. 2001), the method of conjugate directions by Powell, and simplex method, to locate the critical slip surfaces. The Morgenstern and Price method (1965) was used to calculate the factor of safety. Also, Greco (1996) used Spencer's method (1967) in combination with a pattern search and the Monte Carlo technique for the same problem. The comparison of the current results with those obtained by different researchers is summarized in Table 1 . The effect on the factor of safety of using different numbers of slices is also checked. Results from taking 8,16,32,64 and 128 slices are shown in Table 2 . It will be seen from Table 2 that although the value of the minimum factor of safety increases with the number of slices, we find that little improvement in ac-

Table 3. Minimum Safety Factor Given by Minimization Procedures for Example 2

\begin{tabular}{lcc}
\hline Source & Method & $\begin{array}{c}\text { Range of } \\
\text { safety factor }\end{array}$ \\
\hline Yamagami and Ueta (1988) & BFGS & 1.423 \\
& DFP & 1.453 \\
& Powell & 1.402 \\
Greco (1996) & Simplex & 1.405 \\
& Pattern search & 1.400 \\
Malkawi et al. (2001) & Monte Carlo & 1.401 \\
This study & Monte Carlo & 1.330 \\
& Genetic algorithm & 1.395 \\
& & (line and spline) \\
\hline
\end{tabular}

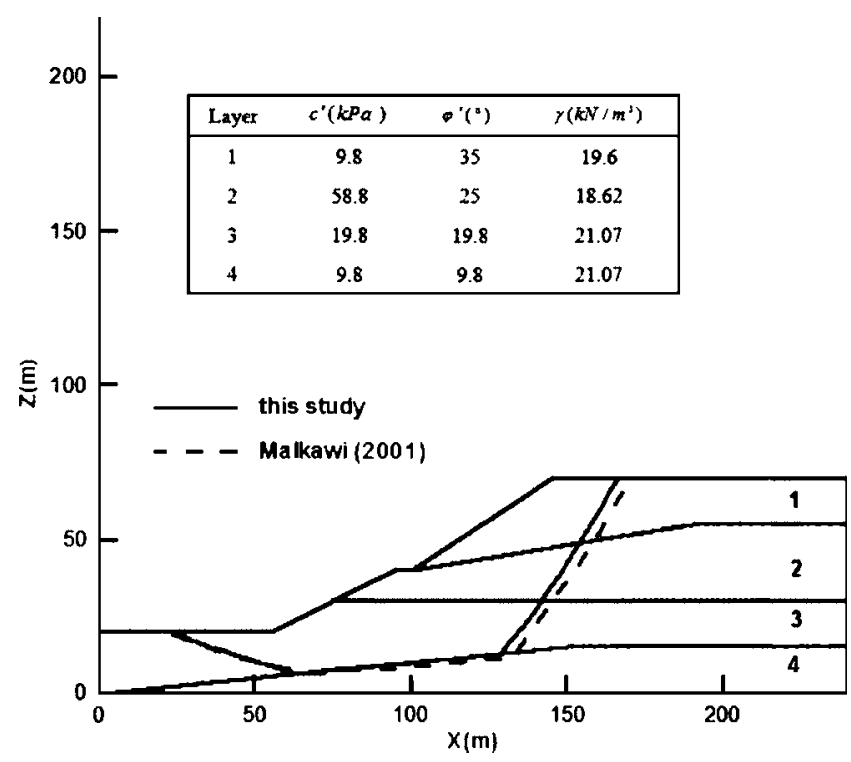

Fig. 5. Cross section of slope of Example 3. The solid line represents the critical slip surface of this study, and the broken line is the solution given by Malkawi et al. (2001, ASCE).

curacy is made when the number of slices is greater than 32 . The next two examples also exhibit similar results and so the number of slices is standardized at 50 .

Because this study focuses on the use of spline curve, Fig. 3 shows the minimum factor of safety for different numbers of nodal points which are connected by spline curves or straight lines. Fig. 3 shows that when the slip surface is connected by spline curves, a convergence to the minimum safety factor is more efficient in terms of the reductive number of nodal points. When the number of nodal points ranges from 4 to 8 , the difference between the computed safety factors is 0.009 for spline curves but 0.046 for straight lines. Comparison of the geometry of the critical slip surfaces (Fig. 2) indicates that 4 nodal points connected by spline curves can generate a critical slip surface similar to that generated by 13 nodal points connected by straight lines (Greco 1996).

\section{Example 2, Multilayered Slope}

This example was also given by Yamagami and Ueta (1988), where a multilayered slope (Fig. 4) is analyzed using the previously described methods. The safety factors were also calculated

Table 4. Minimum Safety Factor Given by Minimization Procedures for Example 3

\begin{tabular}{lcc}
\hline Source & Method & $\begin{array}{c}\text { Range of } \\
\text { safety factor }\end{array}$ \\
\hline Chen and Shao (1988) & Original DFP & $1.011-1.035$ \\
& Modified DFP & $1.009-1.025$ \\
& Steepest descent & 1.025 \\
& Simplex & 1.025 \\
Greco (1996) & Pattern search & $0.973-1.033$ \\
& Monte Carlo & $0.973-0.974$ \\
Malkawi et al. (2001) & Monte Carlo & 0.933 \\
This study & Genetic algorithm & 0.971 (spline, best) \\
& & 0.967 (line, best) \\
\hline
\end{tabular}


using the Morgenstern and Price method. Similarly, Greco (1996) solved this example using Spencer's method and employing pattern search and Monte Carlo techniques. Malkawi et al. (2001) also solved the same example using Spencer's method and Monte Carlo techniques. Table 3 summarizes the results obtained by the present method in comparison with those obtained by different researchers. The critical slip surface (Fig. 4) defined by 4 nodal points connected by spline curves is compared to that defined by 13 nodal points connected by straight lines (Greco 1996). Fig. 3 shows the minimum factors of safety for different numbers of nodal points which are connected by spline curves or straight lines. They also illustrate that the generated slip surface connected by spline curves can reduce the number of nodal points needed to converge to the minimum factor of safety.

\section{Example 3, Slope with Weak Layer}

This example is based on a problem presented by Chen and Shao (1988). The stratigraphy incorporates a thin weak layer below the toe of the slope as shown in Fig. 5. The slope was analyzed using the simplex method, the steepest descent method, the original method of DFP and its modified version. The safety factors are calculated by Spencer's method (1967). Malkawi et al. (2001) also solved the same example using Spencer's method and Monte Carlo techniques. Table 4 summarizes the results obtained by the present method in comparison with those obtained by different researchers. Fig. 3 shows the minimum factors of safety for different numbers of nodal points connected by spline curves or straight lines. The result reveals that the differences between factors of safety from the various methods are insignificant because the critical slip surface is composed of straight lines (Fig. 5). This demonstrates that defining the slip surface using spline curves renders a satisfactory result even though the critical slip surface isn't smooth.

\section{Conclusions}

In this study, a combination of spline curves and genetic algorithm is used to locate the critical slip surface for slope stability calculations. The slip surface defined by straight lines is compared with that defined by spline curves. The results show that critical slip surfaces located by the present study are very close to those identified by other researchers, which also reveal that the present method could be used to analyze the stability of homogenous slopes, multilayered slopes or even slopes involving a weak layer. When the slip surface is defined by spline curves, fewer nodal points are needed to reach the same accuracy, and to generate rational slip surfaces. Thus considerable CPU time and storage memory are saved. This may be especially advantage for complex slopes in large scale engineering problems for which more degrees of freedom are needed.

\section{Acknowledgments}

This research was financially supported by the Major State Basic Research Development Program of China (No. 2002CB412703), and the Knowledge Innovation Project of Chinese Academy of Sciences (No. KJCX2-SW-L1-4).

\section{References}

Baker, R. (1980). "Determination of the critical slip surface in slope stability computations." Int. J. Numer. Analyt. Meth. Geomech., 4(4), 333-359.

Baker, R., and Gaber, M. (1978). "Theoretical analysis of the stability of slopes." Geotechnique, 28(4), 395-411.

Celestino, T. B., and Duncan, J. M. (1981). "Simplified search for noncircular slip surfaces." Proc., 10th Int. Conf. SMFE, Stockholm, Sweden, 391-394.

Chen, Z. Y., and Shao, C. M. (1988). "Evaluation of the minimum factor of safety in slope stability analysis." Can. Geotech. J., 25(4), $735-748$.

Das, S. K. (2005). "Slope stability analysis using genetic algorithm." Electron. J. Geotech. Engrg., 10, 〈http://www.ejge.com/2005/ Ppr0504/Abs0504.htm〉.

Donald, I. B., and Chen, Z. Y. (1997). "Slope stability analysis by the upper bound approach: Fundamentals and methods." Can. Geotech. $J ., 34(6), 853-862$.

Duncan, J. M., and Wright, S. G. (1980). "The accuracy of equilibrium methods of slope stability analysis." Eng. Geol. (Amsterdam), 16(1), $5-17$.

Goh, A. T. C. (1999). "Genetic algorithm search for critical slip surface in multiple-wedge stability analysis." Can. Geotech. J., 36(2), 382-391.

Greco, V. R. (1996). "Efficient Monte Carlo technique for locating critical slip surface." J. Geotech. Engrg., 122(7), 517-525.

Li, K. S., and White, W. (1987). "Rapid evaluation of the critical slip surface in slope stability problems." Int. J. Numer. Analyt. Meth. Geomech., 11(5), 449-473.

Malkawi, A. I. H., Hassan, W. F. and Sarma, S. K. (2001). "Global search method for locating general slip surface using Monte Carlo techniques." J. Geotech. Geoenviron. Eng., 127(8), 688-698.

McCombic, P., and Wilkinson, P. (2002). "The use of the simple genetic algorithm in finding the critical factor of safety in slope stability analysis." Comput. Geotech., 29(8), 699-714.

Morgenstern, N. R., and Price, V. E. (1965). "The analysis of stability of general slip surface." Geotechnique, 15(1), 79-93.

Spencer, E. (1967). "A method of analysis of the stability of embankments assuming parallel interslice forces." Geotechnique, 17(1), $11-26$.

Yamagami, T., and Ueta, Y. (1988). "Search for noncircular slip surfaces by the Morgenstern-Price method." Proc., 6th Int. Conf. on Numerical Methods in Geomechanics, 1219-1223.

Zolfaghari, A. R., Heath, A. C., and McCombie, P. F. (2005). "Simple genetic algorithm search for critical noncircular failure surface in slope stability analysis." Comput. Geotech., 32(3), 139-152. 